\title{
DETERMINATION OF THE THERMAL EXPANSION COEFFICIENT OF NANOSTRUCTURED MATERIALS USING MOLECULAR DYNAMICS
}

\author{
A. R. MUNIZ ${ }^{1}$, L. J. GUARNETTI ${ }^{2}$ e A. F. FONSECA ${ }^{3}$ \\ ${ }^{1}$ Universidade Federal do Rio Grande do Sul, Departamento de Engenharia Química \\ ${ }^{2}$ Universidade Estadual Paulista “Julio de Mesquita”, POSMAT - Programa de Pós-Graduação em \\ Ciência e Tecnologia de Materiais da UNESP, Bauru, Brasil \\ ${ }^{3}$ Universidade Estadual de Campinas, Instituto de Física “Gleb Wataghin”, Departamento de Física \\ Aplicada \\ E-mail para contato: amuniz@enq.ufrgs.br, afonseca@ifi.unicamp.br
}

\begin{abstract}
Carbon nanostructures have attracted the attention from scientists of all areas of knowledge because of their unique combination of mechanical, electronic, optical and thermal properties. The understanding of structure-property relationships is fundamental to the development of innovative practical applications of these materials. One of these properties is the thermal expansion coefficient (TEC). Previous studies have shown that the TEC of some carbon nanostructures is anomalous (negative in a certain range of temperature). In this work, the TEC of carbon nanotubes and graphene are calculated using classical molecular dynamics simulations. Different methodologies were applied to compute this property, in order to investigate the most appropriate for this class of materials. Our results showed good agreement with experimental and other theoretical predictions.
\end{abstract}

\section{INTRODUCTION}

The last two decades were marked by the discovery of new carbon allotropes with characteristic dimensions in the order of nanometers. Carbon nanotubes (Iijima, 1991) and graphene sheets (Novoselov et al., 2004; 2005) are the most important examples of carbon nanostructures. Their unique set of physical and chemical properties has attracted the attention of the scientific community in view of the enormous potential in technological applications.

Amongst the great number of potential applications of carbon nanostructures, ranging from nanoelectronics to reinforced materials (Saito et al., 1998; Baughman et al., 2002; Geim and Novoselov, 2007; Geim and Kim, 2008; Endo et al.; 2008; De Volder et al., 2013), we highlight the recent development of artificial muscles made of carbon nanostructures (Aliev et al., 2009; Sellinger et al., 2010; Hu et al., 2010; Foroughi et al., 2011; Chen et al., 2011; Lima et al., 2012). This system is based on the phenomenon of thermal actuation, which consists in thermal-induced strains capable of performing work on an external system. 


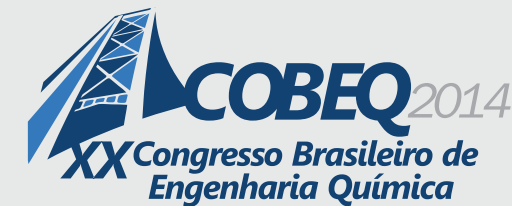

19 a 22 de outubro de 2014

Florianópolis/SC

Although these artificial muscles are macroscopic, the study of the thermal expansion of the individual nanostructures that compose them is the key to understand the phenomenon of thermal actuation. In particular, it is well known that single wall carbon nanotubes and graphene possess negative thermal expansion in a wide range of temperatures (Kwon et al., 2004; Jiang et al., 2004; Kahaly and Waghmare, 2007; Bao et al., 2009; Singh et al., 2010; Yoon et al., 2011; Alamusi et al., 2012). In general, the accurate prediction of the thermal expansion coefficient of carbon nanostructures is of fundamental importance to the description of the thermal behavior and the design of new nanoelectromechanical systems (NEMS) made of these nanostructures.

In the present work, we applied classical molecular dynamics simulations to obtain the TEC of carbon nanostructures, using different approaches. We discuss the suitability, advantages and disadvantages of each methodology. The work is organized as follows. In Section 2, we describe the carbon nanostructures considered in this study, and the different methods used to compute the TEC of the nanostructures. In Section 3, we present the results and in Section 4, we summarize the main conclusions.

\section{NANOSTRUCTURES INVESTIGATED AND COMPUTATIONAL METHODS}

The carbon nanostructures considered in the present study are depicted in Figure 1. We used a $(10,10)$ single wall nanotube (SWCNT) with diameter of $13.56 \AA$ and a length of $100 \AA$. For graphene sheets, five supercells with different sizes were considered (lengths of 46, 62, 67, 135, and $290 \AA$ ). The choice of the value of $100 \AA$ for the nanotube is about twice the one considered in Ref. (Alamusi et al., 2012) and half of that considered in the study of Cao et al., 2005.

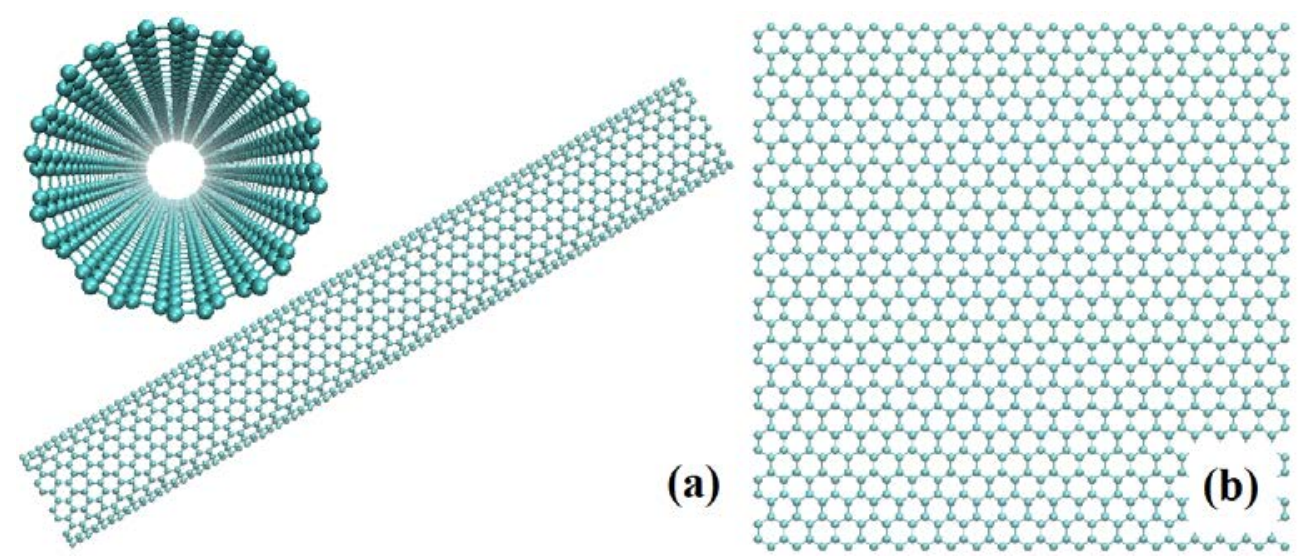

Figure 1 - (a) Upper and side views of a $(10,10)$ single-walled carbon nanotube. (b) Upper view of a graphene sheet.

The linear thermal expansion coefficient $(\alpha)$ of a solid material is given by:

$$
\alpha=\frac{1}{L} \frac{\mathrm{d} L}{\mathrm{~d} T}
$$




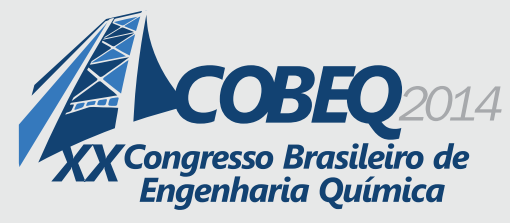

19 a 22 de outubro de 2014
Florianópolis/SC

where $L$ is a characteristic length of the system (nanotube length or graphene supercell length), and $T$ is the absolute temperature. In order to calculate $\alpha$, we need the equilibrium characteristic length of each nanostructure as a function of the temperature $L(T)$. In this study, the function $L(T)$ is obtained by fitting a set of values of lengths $L_{i} \times T_{i}$, corresponding to the equilibrium characteristic lengths of the structure at temperatures $T_{i}$ (in the range of $50 \mathrm{~K}-1000 \mathrm{~K}$ ), to a $4^{\text {th }}$-order polynomial function in $T$.

The values of $L_{i}\left(T_{i}\right)$ are computed using classical MD simulations, according to the second generation of the Reactive Empirical Bond Order (REBO) potential (Brenner, 2002). This interatomic potential has been widely used to describe the structure and estimate the physical properties of carbon allotropes. In all simulations, the Newton's equations of motion were integrated with the velocityVerlet method with a time step of 0.5 fs. Simulations were carried out within the NVT (constant number of atoms, volume and temperature) or NPT (constant number of atoms, pressure and temperature) ensembles. The LAMMPS package (Plimpton, 1995) was used in all simulations.

The use of classical MD simulations allows the study of larger systems, when compared to $a b$ initio methods; however, the accuracy of the results are strongly dependent on the quality of the interatomic potential employed. We have tested three different methods to extract the necessary data from MD simulations to obtain the function $L(T)$ for each structure; they are described in the next subsections.

\subsection{Free edges method (FE)}

In this method, the system is simulated without periodic boundary conditions (system is considered finite) and the MD simulations are performed within the NVT ensemble. The extremities of the structure are passivated with hydrogen atoms, and allowed to move without any constraint. The system has to be long enough to make the effect of edges passivation negligible. Basically, this method consists in performing a series of MD simulations, one for each value of temperature $T_{i}$; after reaching the equilibrium, the characteristic length $L_{i}$ of the structure is taken.

\subsection{Zero pressure method (ZP)}

In this approach, the system is simulated using periodic boundary conditions along all relevant directions (infinite system), and the MD simulations are performed within the NPT ensemble, with pressure set to zero (hydrostatic pressure). The Nosé-Hoover thermostat/barostat was employed in these calculations. In this case, the simulation box is allowed to relax simultaneously with the structure. Because of the periodic boundary conditions, the characteristic lengths $L_{i}$ of the system are the supercell lengths. In this method, for each value of $T_{i}$, the MD simulation must be carried out long enough to ensure full equilibration of the structure, and the average of $L_{i}$ is computed.

\subsection{Lowest energy method (LE)}

In this method, the system is simulated using periodic boundary conditions and the MD simulations are performed within the NVT ensemble. The supercell sizes are fixed and the energy of each simulation is computed after equilibration. For every temperature $T_{i}$, a series of MD simulations 
for different simulation box sizes of the structure is performed; a curve of the computed energies as a function of the box sizes is obtained. Then, we find the length corresponding to the minimum of the energy, that is taken as $L_{i}\left(T_{i}\right)$. This method is well-suited for one-dimensional systems (such as nanotubes), but quite time-consuming for two- and three-dimensional systems.

\section{RESULTS AND DISCUSSION}

\subsection{TEC of the $(10,10)$ SWCNT}

The TEC of the $(10,10)$ SWCNT was calculated from two methods, FE and LE. Figure 2 shows the curves for the SWCNT length versus temperature obtained from both methods.


Figure 2 - SWCNT length versus temperature obtained from FE method (a) and LE method (b). Squares are results from MD simulations and the red line is the polynomial fitting of the points. The difference in the absolute values of the SWNT length between panels (a) and (b) comes from the form of calculation of $L_{i}$. In FE method, the length is obtained by the difference between the coordinates of the carbon atoms close to the SWNT ends. In SE method, the length is obtained as the equilibrium value of the box size, along tube axis.

It is clear from Figure 2 that each approach leads to a different behavior for the thermal expansion of the $(10,10)$ SWCNT, despite using the same method (molecular dynamics) and same interatomic potential (REBO). Several studies have reported that the TEC of SWNTs is negative (Kwon et al., 2004; Jiang et al., 2004; Kahaly and Waghmare, 2007; Alamusi et al., 2012); consequently, results from the FE method should be rejected. One explanation for this discrepancy is given in the study of Cao et al. (2005). According to them, the thermal behavior of SWCNTs comes from three factors: carbon-carbon bond elongation, bending effect of lateral vibration modes and random thermal lattice vibrations. While the carbon-carbon bond distances always increases, because the extremities of the SWCNT are free to move, some bending modes could not be thermally activated. Therefore, the random thermal vibrations are not enough to compensate the expansion of the carbon-carbon bond distances and the length of the SWCNT increases with temperature. 
The linear TEC of the $(10,10)$ SWCNT at $300 \mathrm{~K}$ calculated through the LE method is $\alpha \cong-8,63 \times 10^{-7} \mathrm{~K}^{-1}$ and agrees very well with the results for similar nanotubes studied by Jiang et al. (2004) and Kahaly and Waghmare (2007).

\subsection{TEC of graphene}

The FE method is not suitable to calculate the TEC of graphene because of the same problem with bending modes mentioned for SWNTs. The LE method can be in principle applied, but it is prohibited by the number of simulations required to obtain the values of the supercell sizes corresponding to lowest energies at each temperature. Differently from the SWNT which is a onedimensional structure, graphene is a planar 2D-structure; equilibrium supercell sizes should be computed in two directions. For the SWCNT we needed $N$ simulations, at each temperature, to compute the equilibrium size. For graphene it would be necessary to perform at least $N$ x $N$ simulations. Therefore, we used the ZP method for the calculation of the TEC of graphene, because one (long) simulation is enough for the equilibration of the structure and supercell sizes.

In order to ensure full relaxation of both structure and supercell lengths, each MD simulation in NPT ensemble was run for 1.95 ns (3.9 x $10^{6}$ time steps) per temperature. The values of zigzag and armchair lengths of the graphene sheet $\left(L_{X}\right.$ and $L_{Y}$, respectively), are reported every 100 fs of simulation for calculation of the equilibrium length average. Five supercell sizes were considered in this study: 46, 62, 67, 135, and $290 \AA$. The results for the largest supercell are shown in Figure 3.
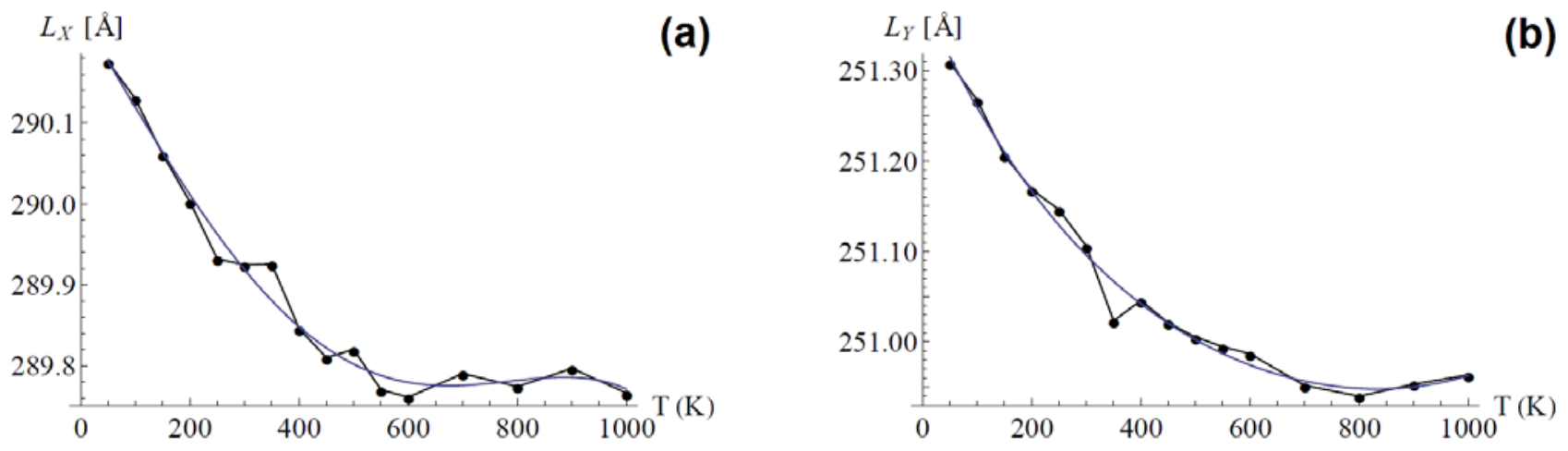

Figure 3-Graphene supercell lengths along zigzag (a) and armchair (b) versus temperature obtained from ZP method. Circles are results from MD simulations and blue line is the polynomial fitting of the points.

Figure 3 shows the variation of the equilibrium lengths $\left(L_{\mathrm{X}}\right.$ and $\left.L_{\mathrm{Y}}\right)$ of the graphene structure as a function of the temperature. For the smaller supercells, we have obtained qualitatively similar results. Figure 4(a) shows the average TEC, $\alpha(T) \equiv 0.5\left(\alpha_{X}(T)+\alpha_{Y}(T)\right)$, of the largest supercell sample calculated using the fitted polynomial functions for $L_{X}(T)$ and $L_{Y}(T)$ and Equation 1 .

Figure 4(b) shows the values of the TEC of graphene at $300 \mathrm{~K}$ obtained for the different supercells. The dependence of the TEC with the supercell size can be clearly seen. For the largest 
supercell investigated, the TEC at $300 \mathrm{~K}$ is of $-2.65 \times 10^{-6} \mathrm{~K}^{-1}$ as predicted by the REBO potential, in reasonable agreement with results from the literature (Mounet and Marzari, 2005; Bao et al., 2009; Singh et al., 2010; Yoon et al., 2011; Sevik, 2014). Although theory of thermal expansion of plates predicts independence of the TEC with size (Schelling and Keblinski, 2003), in case of graphene, it has been shown that low frequency acoustic phonons play an important role in the determination of the negative values of the TEC (Mounet and Marzari, 2005). Supercells have to be made as large as necessary to avoid constraining these low frequency modes. This explains why the TEC of the largest graphene considered here is in reasonable agreement with both $a b$ initio DFT-based theoretical predictions (Mounet and Marzari, 2005; Sevik, 2014) and with experimental results (Bao et al., 2009; Singh et al., 2010; Yoon et al., 2011). Despite the good agreement, calculations for larger supercells are being carried out to verify whether the present results are converged with respect to supercell size.
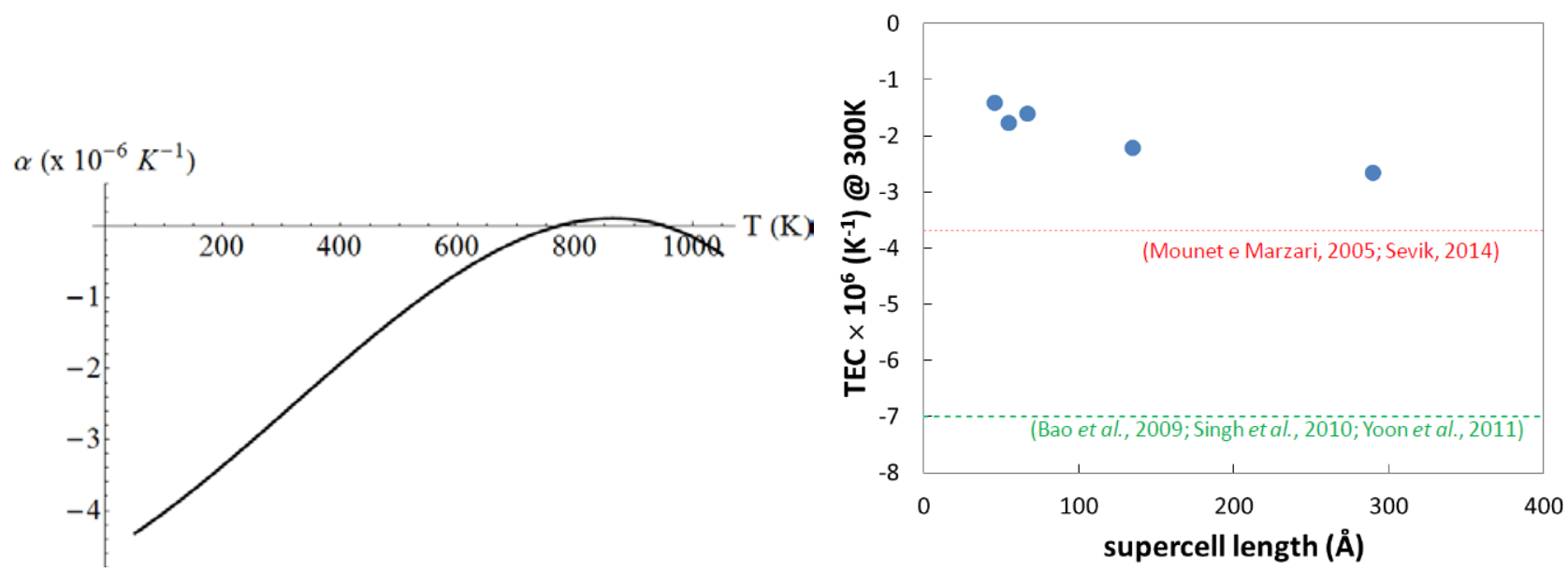

Figure 4-(a) Average TEC of the largest graphene sample as function of T. (b) Computed average TEC of graphene at $300 \mathrm{~K}$ for different supercell sizes. Dotted lines show the values reported in the given references.

\section{CONCLUSIONS}

We have calculated the TEC of a $(10,10)$ SWCNT and graphene using different MD-based methods to obtain the equilibrium values of their characteristic lengths as function of the temperature. We have shown that ZP and LE methods give results in good agreement with the literature. Although the FE method is simpler and less time consuming than the others, the freedom of the edges to move impedes some flexural modes that contribute to the negative value of the TEC of these carbon nanostructures. The results obtained are encouraging, and this methodology is being applied at this moment in the study of thermal expansion of more complex carbon-based nanostructures. Results will be presented in future publications.

\section{ACKNOWLEDGEMENTS}

AFF acknowledges support from CNPq and FAPESP (grant \#2012/10106-8). 


\section{REFERENCES}

ALAMUSI; HU, N.; JIA, B.; ARAI, M.; YAN, C.; LI, J.; LIU, Y.; ATOBE, S.; FUKUNAGA, H. Prediction of thermal expansion properties of carbon nanotubes using molecular dynamics simulations. Computational Materials Science, v. 54, p. 249-254, 2012.

ALIEV, A. E.; OH, J.; KOZLOV, M. E.; KUZNETSOV, A. A.; FANG, S.; FONSECA, A. F.; OVALLE, R.; LIMA, M. D.; HAQUE, M. H.; GARTSTEIN, Y. N.; ZHANG, M.; ZAKHIDOV, A. A.; BAUGHMAN, R. H. Giant-Stroke, Superelastic Carbon Nanotube Aerogel Muscles. Science, v, 323, p. 1575-1578, 2009.

BAO, W.; MIAO, F.; CHEN, Z.; ZHANG, H.; JANG, W.; DAMES, C.; NING LAU, C. Controlled ripple texturing of suspended graphene and ultrathin graphite membranes. Nature Nanotechnology, v. 4, p. 562-566, 2009.

BAUGHMAN, R. H.; ZAKHIDOV, A. A.; DE HEER, W. A. Carbon nanotubes-the route toward applications. Science, v. 297, p. 787-792, 2002.

BRENNER, D. W.; SHENDEROVA, O. A.; HARRISON, J. A.; STUART, S. J.; NI, B.; SINNOT, S. B. A second-generation reactive empirical bond order (REBO) potential energy expression for hydrocarbons. J. Phys.: Condens. Matter, v. 14, p. 783-802, 2002.

CAO, G.; CHEN, X.; KYSAR, J. W. Apparent thermal contraction of single-walled carbon nanotubes. Phys. Rev. B, v. 72, art. n. 235404, 2005.

CHEN, L.; LIU, C.; LIU, K.; MENG, C.; HU, C.; WANG, J.; FAN, S. High-Performance, lowvoltage, and easily-operable bending actuators based on aligned carbon nanotube/polymer composites. ACS Nano v. 5, p. 1588-1593, 2011.

DE VOLDER, M. F. L.; TAWFICK, S. H.; BAUGHMAN, R. H.; HART A. J. Carbon Nanotubes: Present and Future Commercial Applications. Science, v. 339, p. 535-539, 2013.

ENDO, M.; STRANO, M. S.; AJAYAN, P. M. Potential applications of carbon nanotubes. In Carbon Nanotubes. Berlin: Springer-Verlag Berlin, Vol. 111, p. 13-61, 2008.

GEIM, A. K.; NOVOSELOV, K. S. The rise of graphene. Nature Mat. v. 6, p. 183, 2007.

GEIM, A. K.; KIM, P. Carbon Wonderland, Scientific American. [online]: http://www.scientificamerican.com/article/carbon-wonderland/, 2008. (Acessed in: April 15, 2014).

HU, Y.; CHEN, W.; LU, L. H.; LIU, J. H.; CHANG, C. R. Fibrous nanocomposites of carbon nanotubes and graphene-oxide with synergetic mechanical and actuative performance. ACS Nano, v. 4, p. 3498-3502, 2010.

IIJIMA, S. Helical Microtubules of Graphitic Carbon. Nature, v. 354, p. 56-58, 1991.

JIANG, H.; LIU, B.; HUANG, Y.; HWANG, K. C. Thermal Expansion of Single Wall Carbon Nanotubes. J. Engineering Materials and Technology, v. 126, p. 265-270 2004.

KAHALY, M. U.; WAGHMARE, U. V. Size dependence of thermal properties of armchair carbon nanotubes: A first-principles study. Appl. Phys. Lett., v. 91, art. n. 023112, 2007. 
KWON, Y. -K.; BERBER, S.; TOMÁNEK, D. Thermal Contraction of Carbon Fullerenes and Nanotubes. Phys. Rev. Lett., v. 92, art. n. 015901, 2004.

FOROUGHI, J.; SPINKS, G. M.; WALLACE, G. G.; OH, J.; KOZLOV, M. E.; FANG, S.; MIRFAKHRAI, T.; MADDEN, J. D. W.; SHIN, M. K.; KIM, S. J.; BAUGHMAN, R. H. Torsional Carbon Nanotube Artificial Muscles. Science, v. 334, p. 494-497, 2011.

LIMA, M.D.; NA, LI; ANDRADE, M. J.; FANG, S.; JIYOUNG, OH; SPINKS, G. M.; KOZLOV, M. E.; HAINES, C. S.; SUH, D.; FOROUGHI, J.; KIM, S. J.; CHEN, Y.; WARE, T.; SHIN, M. K.; MACHADO, L. D.; FONSECA, A. F.; MADDEN, J. D. W.; VOIT, W. E.; GALVÃO, D. S.; BAUGHMAN, R. H. Electrically, Chemically, and Photonically Powered Torsional and Tensile Actuation of Hybrid Carbon Nanotube Yarn Muscles. Science, v. 338, p. 928-932, 2012.

MOUNET, N.; MARZARI, N. First-principles determination of the structural, vibrational and thermodynamic properties of diamond, graphite, and derivatives. Phys. Rev. B, v. 71, art. n. 205214, 2005.

NOVOSELOV, K. S.; GEIM, A. K.; MOROZOV, S. V.; JIANG, D.; ZHANG, Y.; DUBONOS, S. V.; GRIGORIEVA, I. V.; FIRSOV, A. A. Electric Field Effect in Atomically Thin Carbon Films. Science, v. 306, p. 666-669, 2004.

NOVOSELOV, K. S.; JIANG, D.; SCHEDIN, F.; BOOTH, T. J.; KHOTKEVICH, V. V.; MOROZOV, S. V.; GEIM, A. K. Two-dimensional atomic crystals. Proc. Natl. Acad. Sci. USA, v. 102, p. 10451-10453, 2005.

PLIMPTON, S. Fast Parallel Algorithms for Short-Range Molecular Dynamics. J. Comp. Phys., v. 117, p. 1-19 1995.

SAITO, R.; DRESSELHAUS, G.; DRESSELHAUS, M. S. Physical Properties of Carbon Nanotubes. London: Imperial College Press, 1998.

SCHELLING, P. K.; KEBLINSKI, P. Thermal expansion of carbon structures. Phys. Rev. B, v. 68, art. n. 035425, 2003.

SELLINGER, A. T.; WANG, D. H.; TAN, L. -S.; VAIA, R. A. Electrothermal polymer composite actuators. Adv. Mater., v. 22, p. 3430-3435, 2010.

SEVIK, C. Assessment on lattice thermal properties of two-dimensional honeycomb structures: Graphene, h-BN, h-MoS2, and h-MoSe2. Phys. Rev. B v. 89, 035422, 2014.

SINGH, V.; SENGUPTA, S.; SOLANKI, H. S.; R. DHALL, R.; A. ALLAIN, A.; DHARA, S.; PANT, P.; DESHMUKH, M. M. Probing thermal expansion of graphene and modal dispersion at low-temperature using graphene nanoelectromechanical systems resonators. Nanotechnology, v. 21, art. n. 165204, 2010.

YOON, D.; SON, Y. -W.; CHEONG, H. Negative Thermal Expansion Coefficient of Graphene Measured by Raman Spectroscopy. Nano Letters, v. 11, p 3227-3231, 2011. 